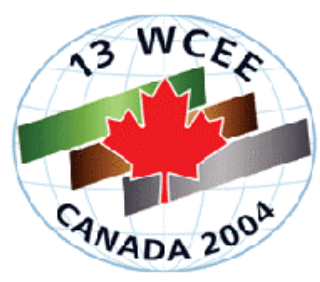

$13^{\text {th }}$ World Conference on Earthquake Engineering

Vancouver, B.C., Canada

August 1-6, 2004

Paper No. 789

\title{
EFFECTIVENESS OF CABLE-RESTRAINER FOR MITIGATING ROTATION OF A SKEWED BRIDGE SUBJECTED TO STRONG GROUND SHAKING
}

\author{
Gakuho WATANABE ${ }^{1}$ and Kazuhiko KAWASHIMA ${ }^{2}$
}

\begin{abstract}
SUMMARY
Skewed bridges collapsed due to unseating of the decks from their support resulted from rotation of the decks around the vertical axis in past earthquakes. Skewed bridges show very unique structural response as a result of poundings of the decks to the substructures and the effect of restrainers. This paper clarifies the mechanism of rotations of skewed bridges and shows the effectiveness of cable restrainers on the mitigation of the rotations. Three configurations of cable restrainers are clarified here: 1) cable restrainers in the weak axis, 2) cable restrainers in the longitudinal direction, and 3) cable restrainers crossing the ends of adjacent decks. It is found that the cross cable restrainers are effective on the mitigation of the rotation of skewed bridges.
\end{abstract}

\section{INTRODUCTION}

It is well known that skewed bridges show a very unique structural response under a strong ground excitation. A deck rotates around the vertical axis when the deck collides with the abutment as shown in Fig. 1. When a deck rotates with an angle $\varphi$, the relative displacements of the deck at an acute edge and an obtuse edge, $u_{a}$ and $u_{o}$, respectively, are

$$
u_{a}=\ell_{a} \varphi \sin \theta_{a} ; u_{o}=\ell_{o} \varphi \sin \theta_{o}
$$

where $\ell_{a}$ and $\ell_{o}$ : diagonal length between acute edges and obtuse edges, respectively, $\theta_{a}$ and $\theta_{o}$ : angle between axial direction and acute direction, and angle between axial direction and obtuse direction, respectively. As a consequence of the rotation, the supported length of deck by the abutments decreases with an amount of $u_{a}$ and $u_{o}$ at acute edges and obtuse edges, respectively, and this often resulted in unseating of skewed bridges in past earthquakes. Because $u_{a}>u_{o}$, an unseating generally starts to occur at the acute edges.

Many studies have been conducted on the seismic response of skewed bridges and the effect of restrainers. For example, Chen and Penzien (1975) analyzed the effect of poundings between a deck and an abutment on the seismic response of a short-span skewed bridge. Liu et al (1990) showed that the strut action of slabs results in a rotation of a skewed bridge. In a continuous bridge with intermediate hinges,

\footnotetext{
${ }^{1}$ Research Associate, Tokyo Institute of Technology, Tokyo, Japan. Email: gappo@cv.titech.ac.jp

${ }^{2}$ Professor, Tokyo Institute of Technology, Tokyo, Japan. Email: kawasima@cv.titech.ac.jp
} 


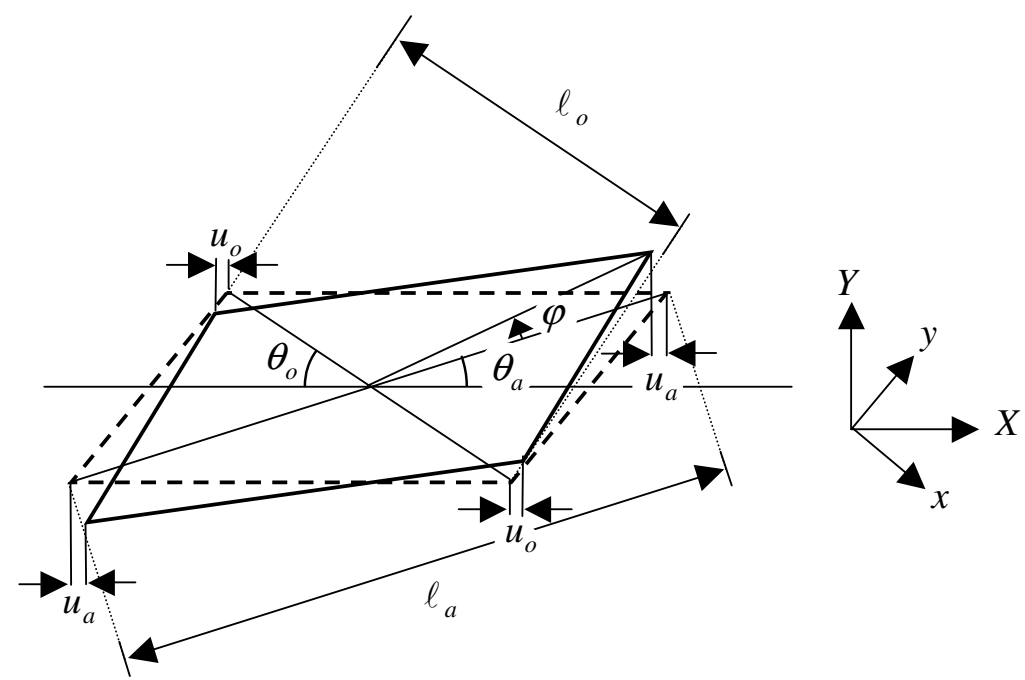

Fig.1 Decrease of Length Supported by Abutment due to Rotations of Deck

excessive relative displacement tends to be accumulated at the hinges under a strong excitation. Enough seat length or adequate strength of restrainers must be maintained in seismic design of bridges. Saiidi et al. (1996) conducted a parametric study on the effect of hinge restrainers on the seismic response of multiple-frame bridges, and suggested an adequate restrainer slack size. Trochalakis et al. (1997) proposed a method to estimate the displacement response at a hinge from the pseudo displacement spectra. Shoji and Kawashima evaluated the effects of shock absorbers on the mitigation of the deck displacement at hinges. DesRoches and Fenves (2000) proposed a design method for the restrainers at intermediate hinges to limit the hinge displacement to a specified level.

Because restrainers configuration which is effective to reduce the risk of unseating has not yet been analyzed in the past studies, this paper focuses on the effectiveness of cable restrainers on the mitigation of rotation of a skewed bridge.

\section{MECHANISM OF ROTATIONS OF SKEWED BRIDGES}

\section{Effect of the Impact Forces}

To distinguish the direction of skew from the bridge axis (longitudinal direction, $\mathrm{X}$ direction) and transverse direction ( $\mathrm{Y}$ direction), the direction parallel to the ends of skewed bridge and the direction perpendicular to this direction are referred hereinafter as skewed transverse direction (y direction) and skewed longitudinal direction (x direction), respectively. When a deck collides with an abutment as shown in Fig. 2, a moment $M_{I}$ develops around the center of gravity as

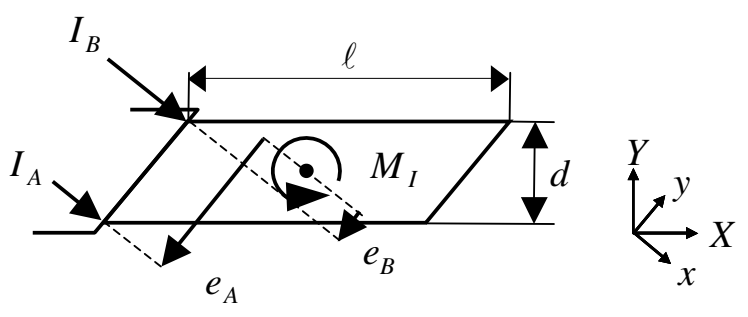

Fig.2 Rotational Moment due to Impact Force 


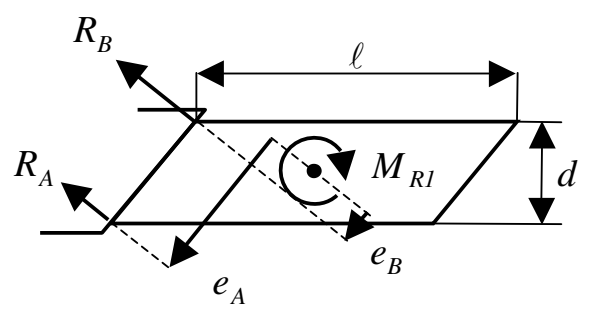

(a) Type 1

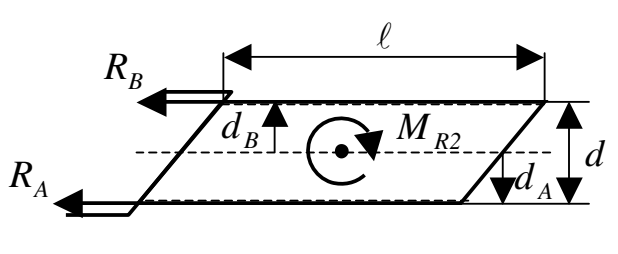

(b) Type 2
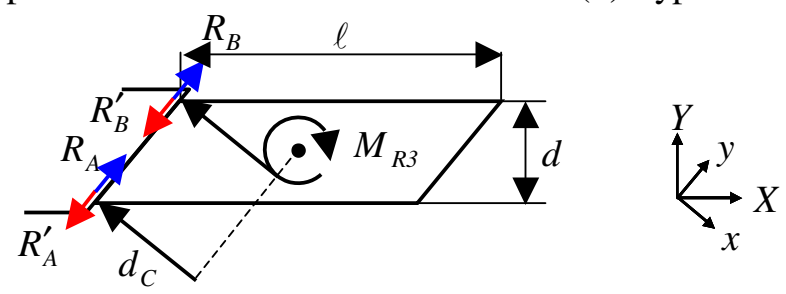

(c) Type 3

Fig.3 Rotation due to Restrainer Force

$$
M_{I}=I_{A} e_{A}+I_{B} e_{B}
$$

where $I_{A}$ and $I_{B}$ represent impact forces at the acute and obtuse edges, respectively, in the skewed longitudinal direction, and $e_{A}$ and $e_{B}$ represent the eccentricity for $I_{A}$ and $I_{B}$ from the mass center, respectively, given as

$$
\begin{aligned}
& e_{A}=\left(\ell \cos \theta+\frac{d}{\tan \theta}\right) / 2 \\
& e_{B}=\left(\ell \cos \theta-\frac{d}{\tan \theta}\right) / 2
\end{aligned}
$$

where $\theta$ represents the skew angle, and $\ell$ and $d$ represent the length and width of the deck in the longitudinal and transverse directions, respectively.

\section{Effect of Restrainers}

When restrainers are provided in the direction which results in an eccentricity from the mass center of the deck, a rotation occurs in the skewed bridge. Although there are many ways of providing restrainers, considered here are cable restrainers positioned in three ways: (1) two cable restrainers are provided along the skewed longitudinal direction (x direction) at both sides (Type 1), (2) two cable restrainers are provided along the longitudinal direction (X direction) at both sides (Type2), and (3) two cable restrainers each (four restrainers in total) are provided along the skewed transverse direction (y direction) at both ends (Type 3). Type 3 restrainers intend to prevent rotations of the decks by restricting relative movements between adjacent decks in the skewed transverse direction. However the movement in the skewed longitudinal direction is not restricted by the type 3 restrainers.

As shown in Fig. 3, the moments which are induced at the mass center by types 1, 2 and 3 restrainers, $M_{R 1}, M_{R 2}$ and $M_{R 3}$, respectively, are

$$
\begin{aligned}
& M_{R 1}=-\left(R_{A} e_{A}+R_{B} e_{B}\right) \\
& M_{R 2}=-\left(R_{A}-R_{B}\right) \cdot d / 2
\end{aligned}
$$




$$
M_{R 3}=\left\{\begin{array}{cc}
\left(R_{A}+R_{B}\right) \cdot d_{C} & \text { for counter clockwise rotation } \\
-\left(R_{A}^{\prime}+R_{B}^{\prime}\right) \cdot d_{C} & \text { for clockwise rotation }
\end{array}\right.
$$

where $R_{A}$ and $R_{B}$ : tensions in two restrainers when the bridge rotates counterclockwise, $R_{A}^{\prime}$ and $R_{B}^{\prime}$ : tensions in two restrainers when the bridge rotates clockwise, and $d_{c}=\ell / 2 \cdot \sin \theta$. If $R_{A}=R_{B}=R, M_{R 1}$, $M_{R 2}$ and $M_{R 3}$ by Eqs. (4), (5) and (6) become

$$
\begin{gathered}
M_{R 1}=R \ell \cos \theta \\
M_{R 2}=0 \\
M_{R 3}=R \ell \sin \theta
\end{gathered}
$$

Consequently, $M_{R 1}=0.64 R \ell, M_{R 2}=0$ and $M_{R 3}=0.76 R \ell$ at a skew angle $\theta=50$ degree. Since $R_{A}$ is not necessarily equal to $R_{B}$ in reality, Eqs. (7), (8) and (9) provide only an approximate estimate on the magnitude of the moments induced in the skewed bridge by restrainers.

\section{Effect of incoherent response of substructures}

If the seismic responses of substructures are not identical, a relative displacement occurs between the substructures, which results in a rotation of the deck as shown in Fig. 4. The incoherent response of substructures occurs when stiffness of the substructures is not identical or when the bridge is subjected to a spatially differential ground motion. Representing the displacement at the both ends of the deck in the skewed transverse direction (y direction) as $u_{p i}$ and $u_{P j}$, the rotation of the deck is written as

$$
\varphi=\frac{\left(u_{P i}-u_{P j}\right) \cdot \cos \theta}{\ell}
$$
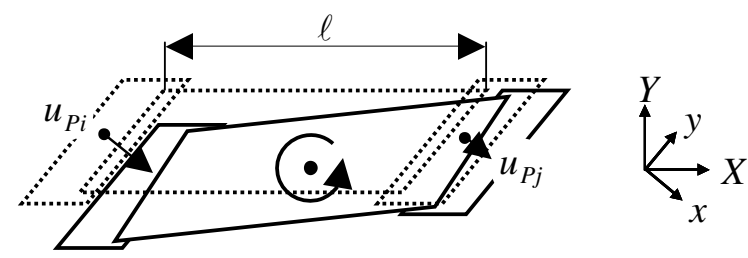

Fig.4 Rotation due to Incoherent Response

\section{TARGET BRIDGE AND ANALYTICAL BRIDGE MODEL}

An analysis was conducted on a $200 \mathrm{~m}$ long skewed bridge with a skewed angle $\theta$ of 50 degrees as shown in Fig. 5. The bridge consists of a 3 span continuous deck (deck 1), 2 simply supported decks (deck 2 and 3), two abutments and four columns. The decks are supported by fixed or movable bearing plate bearings.

Fig. 6 shows a three dimensional discrete model. The deck was idealized by a beam with the equivalent flexural and torsional stiffness. The strut action of deck was idealized by rigid lateral beams. The soilstructure interaction effect was idealized by a set of translational and rotational elastic springs. Because the bearing plate bearings were designed in accordance with the pre-1995 Kobe earthquake code, it is likely that they suffer extensive damage when the bridge is subjected to a strong near-field ground motion. Consequently, both the fixed and movable bearings were idealized by a friction type hysteretic model with 


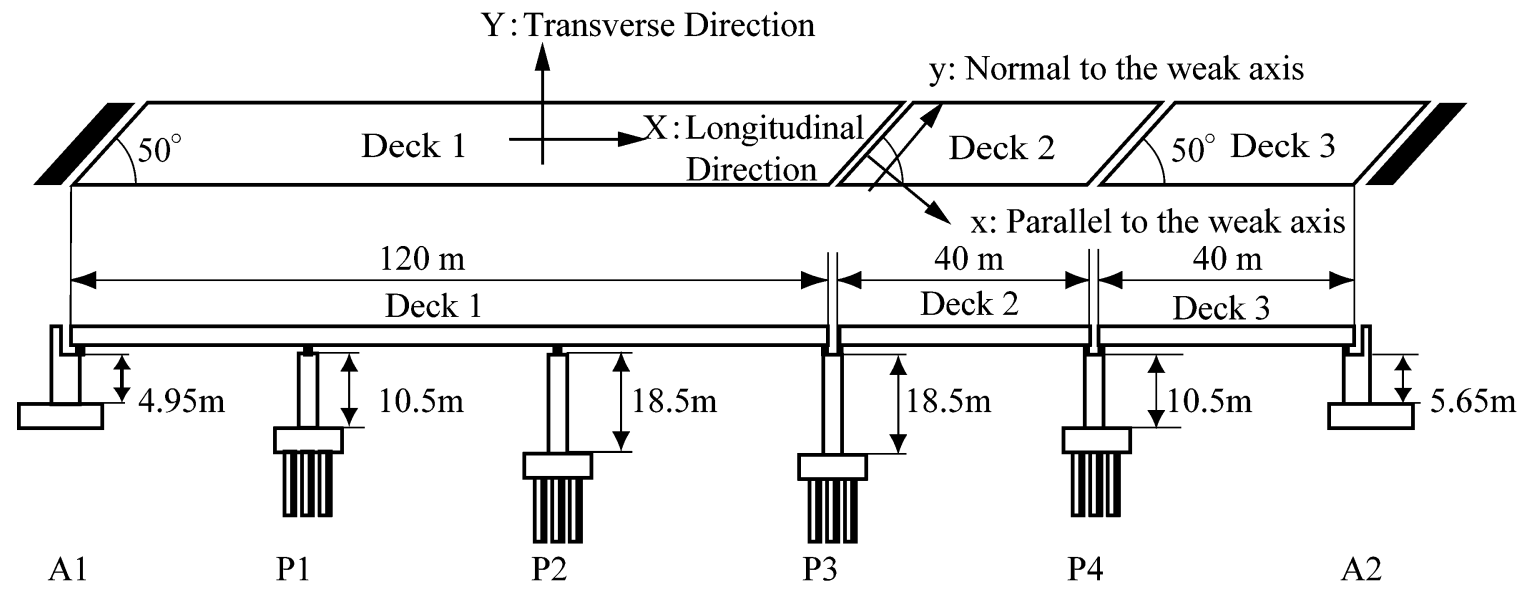

Fig.5 Target Bridge

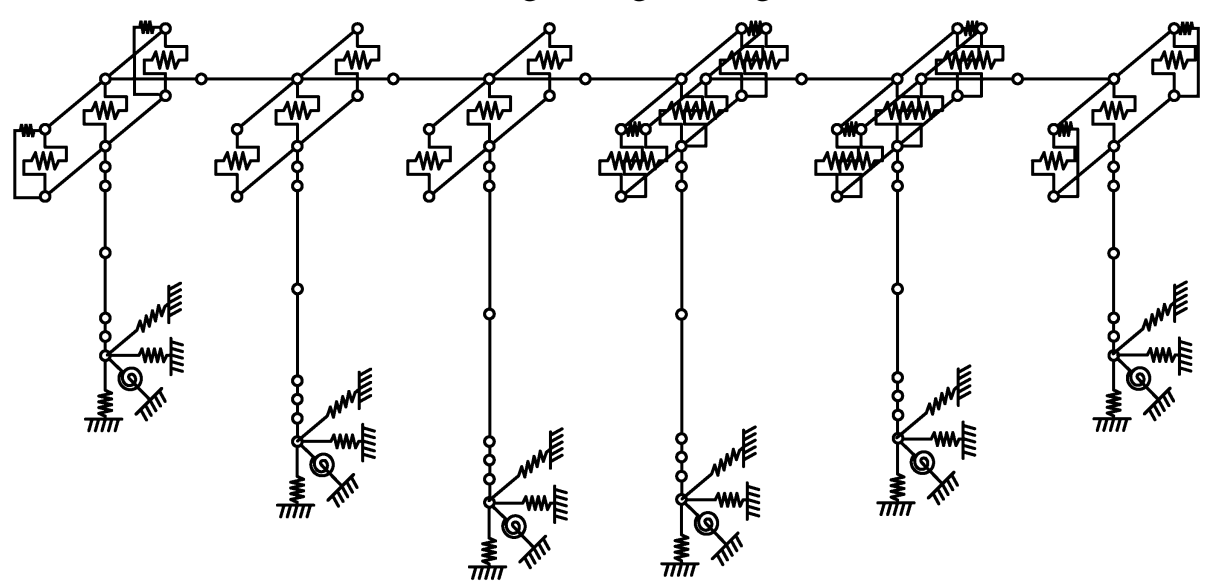

Fig. 6 Analytical Model

a friction coefficient of 0.05 . The plastic deformation of the columns at the plastic hinge was disregarded for simplicity.

The design force of cable restrainers $H_{F}$ was determined as

$$
H_{F}=\alpha \cdot W / m
$$

where, $W$ : weight of a deck, $\alpha$ : factor of safety, and $m$ : number of cables per deck. Based on the Japanese seismic design code, $\alpha$ was set 1.5. Since the weight of decks 1,2 and 3 is $13.55 M N, 4.52 M N$ and 4.52 MN, respectively, the design force $H_{F}$ is $5.08 \mathrm{MN}$ at deck 1 and $1.70 \mathrm{MN}$ at decks 2 and 3 by assuming $m=4$. Since it is not appropriate to use different design forces in a same bridge, 5.081 $M N$ was used here for all cable restrainers. Consequently, a PC cable strands (SWPR19) with the first and second yields of 7.23 $M N$ and $8.07 M N$, respectively, was used for a cable restrainer. Assuming the restrainers are $2 \mathrm{~m}$ long, the first and second yield displacements $\left(u_{1}\right.$ and $\left.u_{2}\right)$ are $15.4 \mathrm{~mm}$ and $30 \mathrm{~mm}$, respectively. Restrainers resist only tension and their force vs. relative displacement hysteresis is idealized as shown in Fig. 7. Since a pair of restrainers is set at each end in type 3, they resist both positive and negative relative displacement $\Delta u$.

Poundings between the decks and between the decks and two abutments were idealized by the impact spring as 


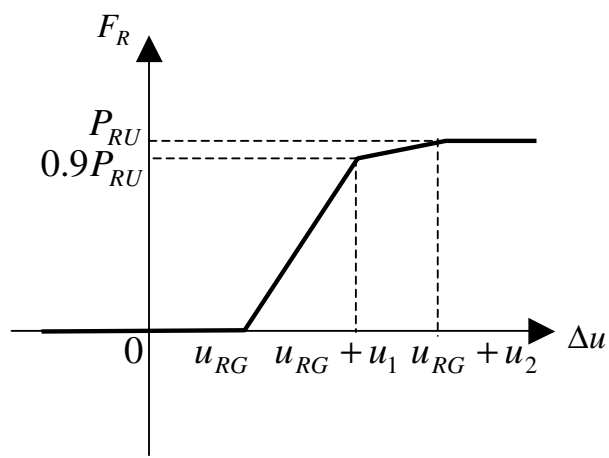

(a) Type 1 and 2

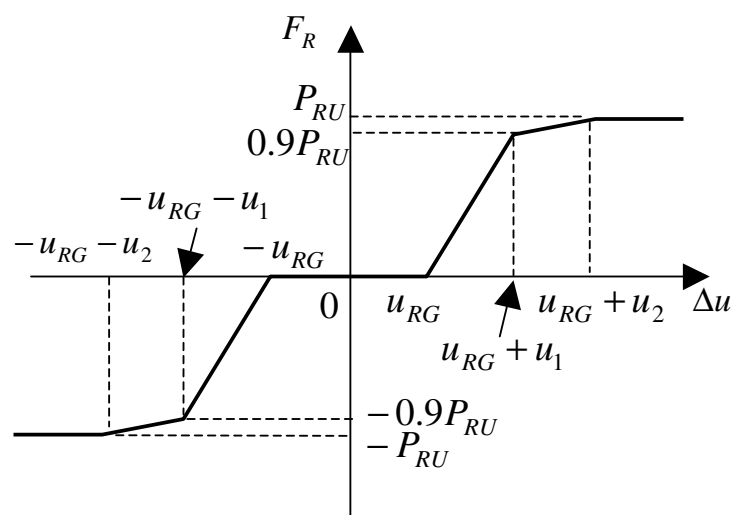

(b) Type 3

Fig.7 Idealization of Restrainers

$$
P_{I}=k_{I}\left\langle u+\Delta_{G}\right\rangle\left(u+\Delta_{G}\right)
$$

where

$$
\left\langle u+\Delta_{G}\right\rangle=\left\{\begin{array}{l}
1 \cdots u+\Delta_{G}<0 \\
0 \cdots u+\Delta_{G} \geq 0
\end{array}\right.
$$

in which $u$ and $\Delta_{G}$ : relative displacement and magnitude of gap at four joints, respectively, and $k_{I}:$ the stiffness of the impact spring. The impact spring stiffness $k_{I}$ was set $9.8 \mathrm{GN} / \mathrm{m}$ so that a parameter $\gamma$ defined as

$$
\gamma=\frac{k_{I} L}{n E A}
$$

was nearly equal to 1.0 (Kawashima and Penzien 1979, Watanabe and Kawashima 2004), in which $E, A$ and $L$ : elastic modulus, section and length of the deck, and $n$ : number of beam element per deck.

The bridge was subjected to NS and EW components of a ground acceleration recorded at JMA Kobe Observatory during the 1995 Kobe earthquake as shown in Fig. 8.

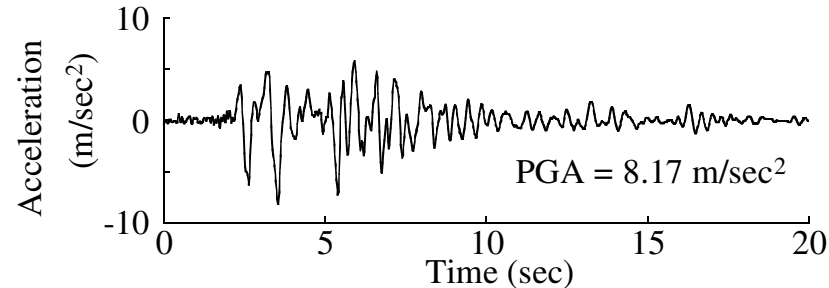

(a) NS Component

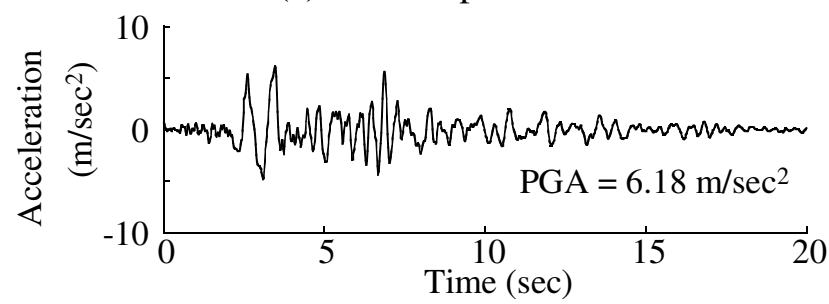

(b) EW Component

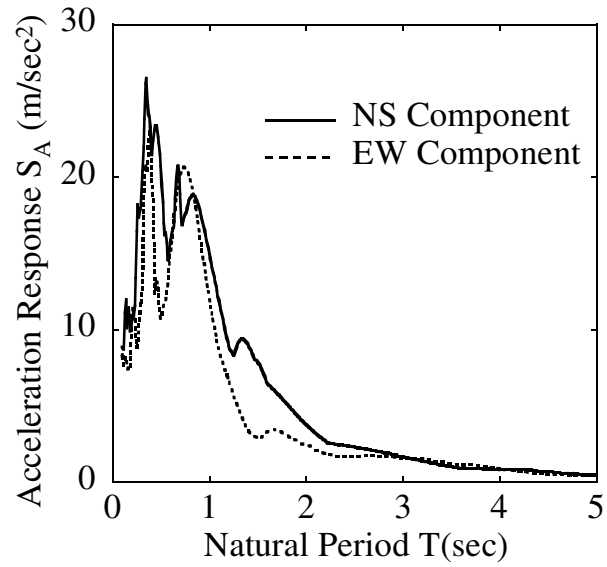

(c) Response Acceleration ( $\xi=0.05$ )

Fig. 8 Accelerations and Response Acceleration of the JMA Kobe Record 


\section{EFFECT OF POUNDINGS AND RESTRAINERS}

\section{Type 1 restrainers}

Fig. 9 shows how three decks displace, rotate and collide among the three decks and the two abutments when type 1 restrainers were provided. Response during the first $2.74 s$ is presented here. The gap of the restrainers $\Delta_{G}$ is set $50 \mathrm{~mm}$ in Eqs. (12) and (13). Fig. 10 shows relative displacements, impact and

Abuttment 1

Abuttment 2

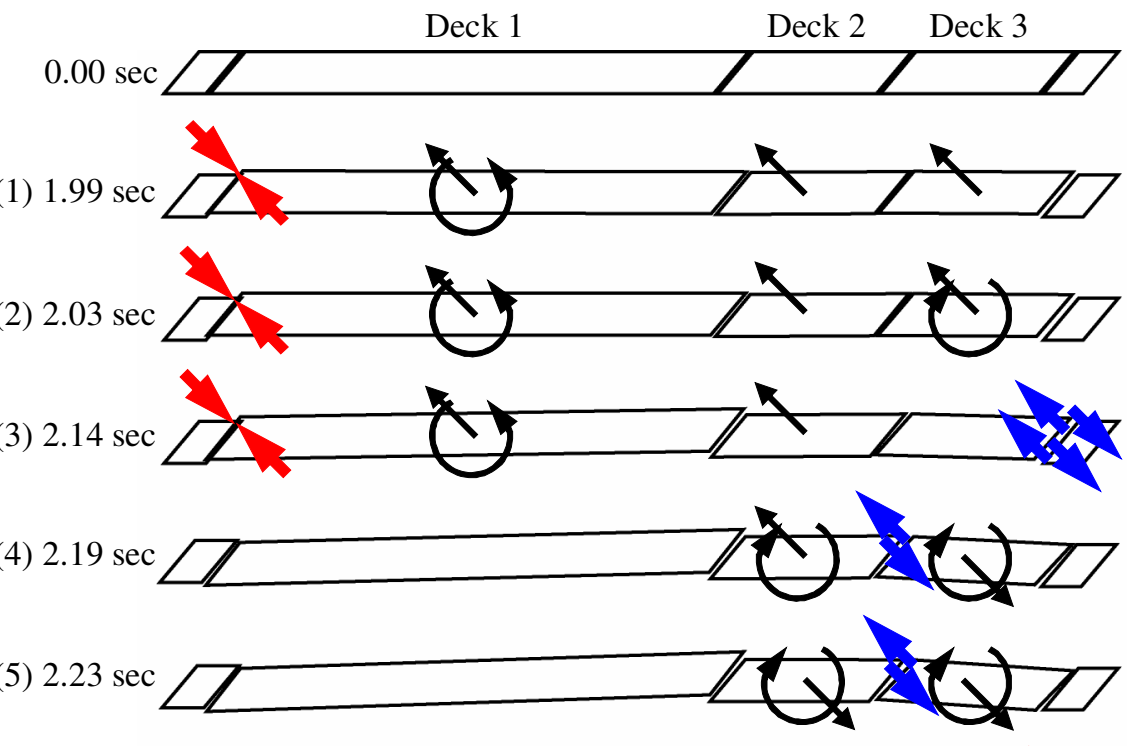

(6) $2.38 \mathrm{sec}$

(7) $2.34 \mathrm{sec}$

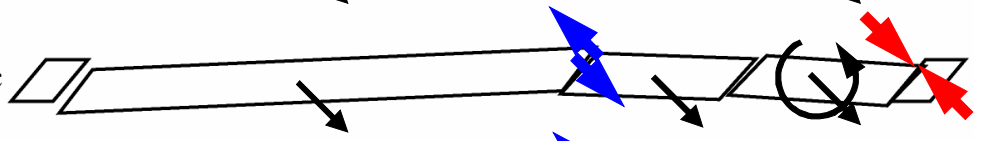

(8) $2.46 \mathrm{sec}$

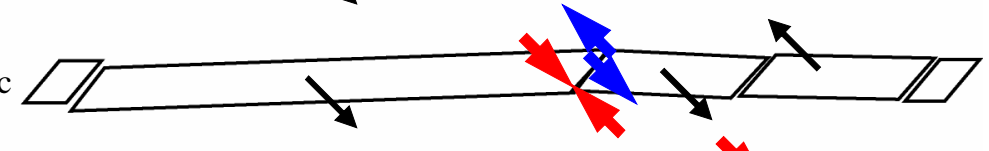

(9) $2.52 \mathrm{sec}$

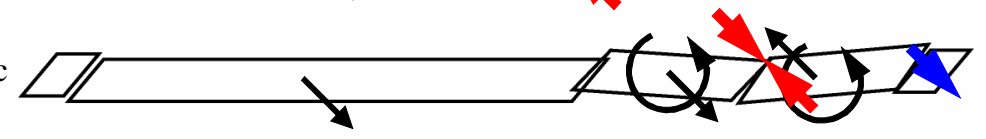

(10) $2.59 \mathrm{sec}$

(11) $2.74 \mathrm{sec}$
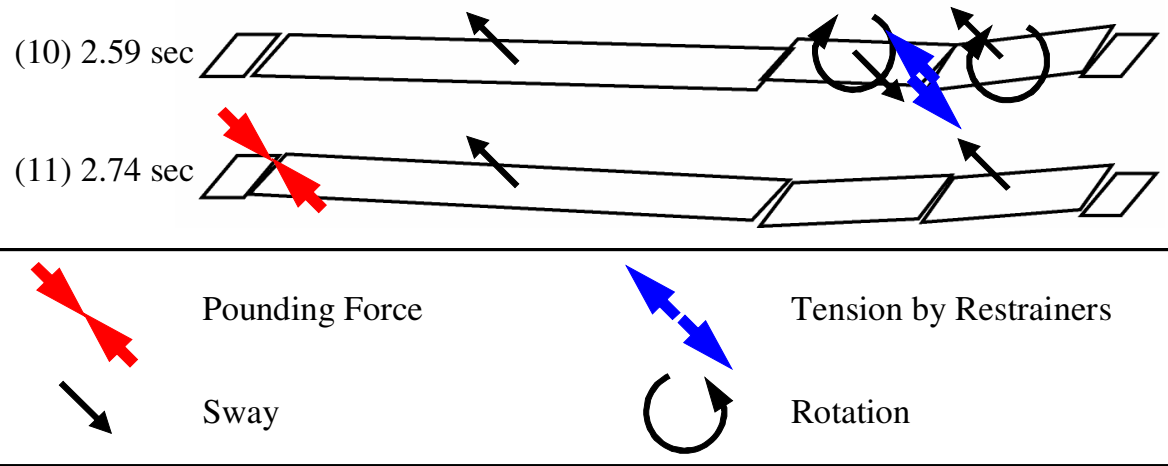

Fig.9 Deck Displacement during the First 2.74 second 


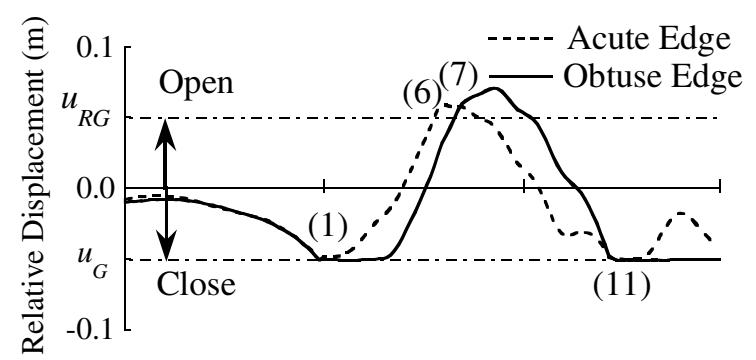

(a) Relative Displacement between Abutment and Deck 1 in y Direction

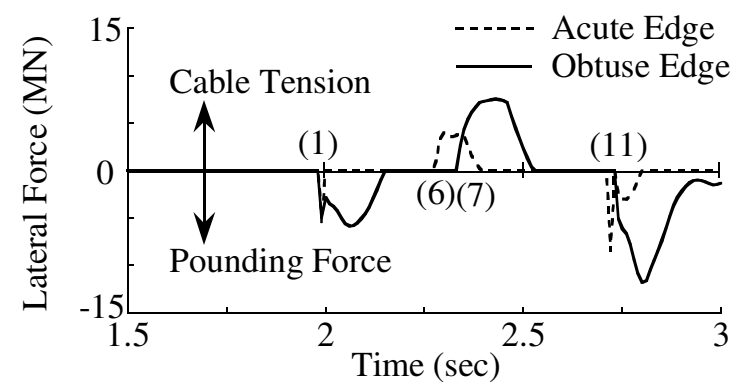

(b) Impact Force and Restrainers Force between Abutment 1 and Deck 1

(1) Left End

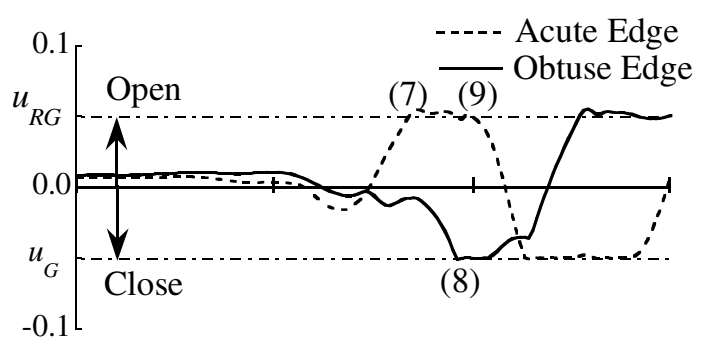

(a) Relative Displacement between Deck1 and Deck 2 in y Direction

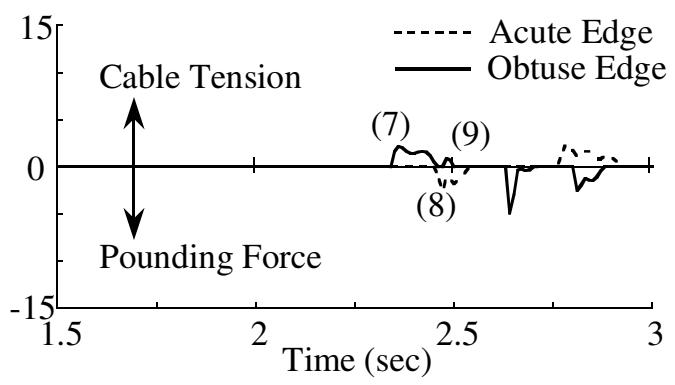

(b) Impact Force and Restrainers Force between Decks 1 and 2

(2) Right End

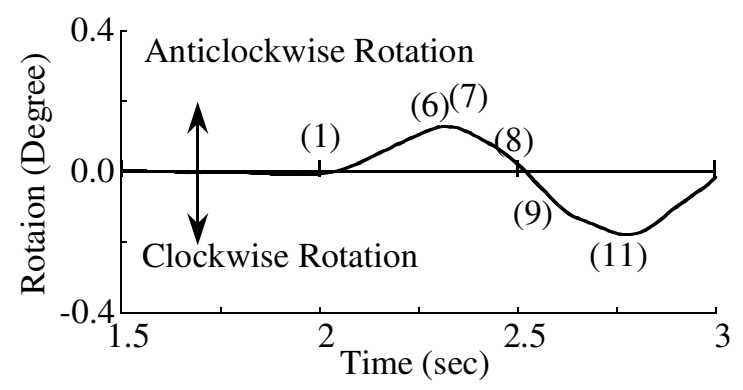

(3) Rotation

Fig. 10 Response of Deck 1 with Type 1 Restrainers

restrainers forces, and the rotation of deck 1. The numbers in parenthesis in Fig. 10 correspond to the numbers in Fig. 9.

The deck 1 first collides with the abutment 1 at $1.99 s$ (No. 1, refer to Fig. 9). This collision continues until $3.13 s$ with the peak impact force of $6 M N$, which corresponds to $45 \%$ of the weight of deck 1 of 13.55 $M N$. This collision results in an anticlockwise rotation of the deck 1 (refer to Fig. 9(3)) depending on the mechanism shown in Fig. 2. The anticlockwise rotation then results in separation of the deck 1 from the abutment 1 . When the separation reaches the gap $\Delta_{G}$, the restrainer at the acute edge first starts to resist further separation at $2.28 s$ (No. 6) and the restrainer at the obtuse edge follows this at $2.34 s$ (No. 7). The restrainers at the obtuse and acute edges continue to pull the deck 1 until $2.39 s$ and $2.59 s$ with the maxim force of 3.8 MN and 7.5 MN, respectively. As shown in Fig. 10, this action by restrainers changes the anticlockwise rotation to a clockwise rotation (No. 7). At the instance of $2.34 s$ (No. 7), the restrainer between the acute edge of deck 1 and the obtuse edge of the deck 2 starts to reist with the maximum force of $2.3 \mathrm{MN}$. Furthermore, the obtuse edge of the deck 1 collides with the acute edge of the deck 2 at $2.52 \mathrm{~s}$ (No. 9), which results in the maximum impact force of $2.5 \mathrm{MN}$. Those two actions accelerate the clockwise 


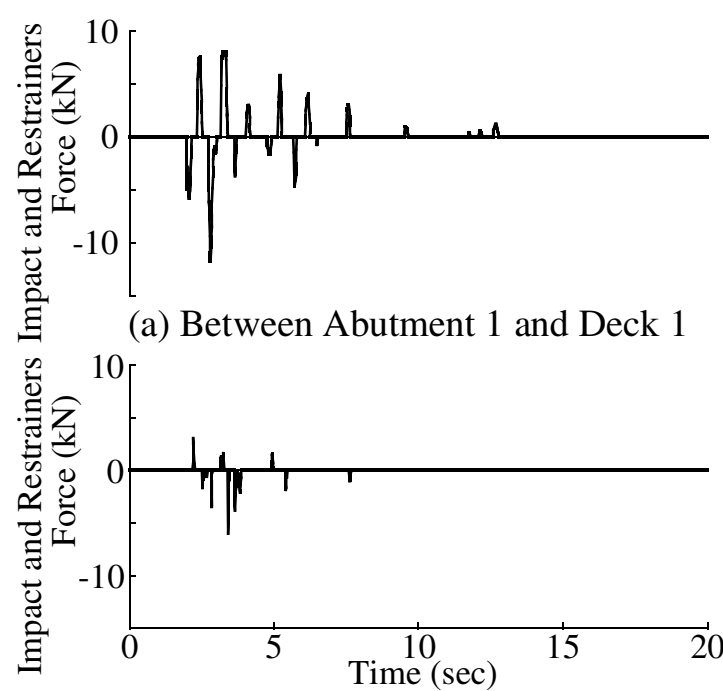

(c) Between Deck 2 and Deck 3

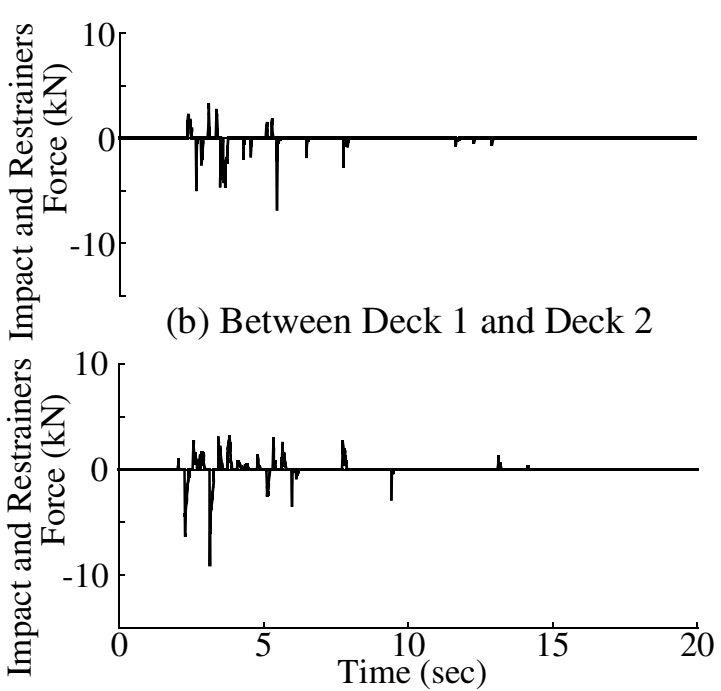

(d) Betweem Deck 3 and Abutment 2

Fig. 11 Impact Force and Restrainers Force between Adjacent Deck Components

rotation of the deck 1 . In the similar manner, complex responses continue resulting from actions of restrainers and collisions. An action results in another action, thus responses occur progressively.

Fig. 11 shows the force induced by poundings (compression) and restrainers (tension) at one of the two sides of the decks. The force induced at the other side of the deck is not presented here due to space limitation. The maximum impact force reached $12 M N$ between the deck 1 and the abutment 1 . Because the weight of deck 1 is $13.55 \mathrm{MN}$, this impact force corresponds to $89 \%$ of the weight of deck 1 . On the other hand, the maximum restrainer force was $8.1 \mathrm{MN}$ between deck 1 and abutment 1 . Since the deck 1 is a three span continuous, larger impact force and restrainer force developed between the deck 1 and the abutment 1 .

Fig. 12 shows rotation response of three decks. The results computed for other gaps $\Delta_{G}$ of $150 \mathrm{~mm}$ and $300 \mathrm{~mm}$ are also presented here for comparison. The maximum rotations are in the range of -0.2 and 0.6 degree at $\Delta_{G}$ of $50 \mathrm{~mm}$. The rotations of the decks increase as the gap $\Delta_{G}$ increases. It is noted that the deck 2 and 3 rotates clock wisely while the deck 1 rotates anticlockwise. This is because three decks displace as shown in Fig. 13 at the instance of the peak response displacement. Fig. 14 shows a graphical sketch of the displacement modes. The deck displacement of the skewed bridge with type 2 and 3 restrainers, which will be described later, are also presented in Figs. 13 and 14 for comparison

\section{Type 2 or 3 restrainers}

Fig. 15 shows rotation response of three decks when type 2 restrainers are equipped. Rotation of the deck 3 reached over 0.8 degree, which is much larger than the rotations of the decks with type 1 restrainers. This is because the moment around the mass center $M_{R 2}$ is smaller than $M_{R 1}$ as shown in Eqs. (7) and (8). Smaller moment by the type 2 restrainers resulted in larger deck rotation, in particular at the deck 3. It is also noted in Fig. 15 that rotations of the three decks occurred only anticlockwise. From Figs. 13 and 14, it is observed larger relative displacement in the skewed direction occurred between the deck 1 and the abutment 1 . 


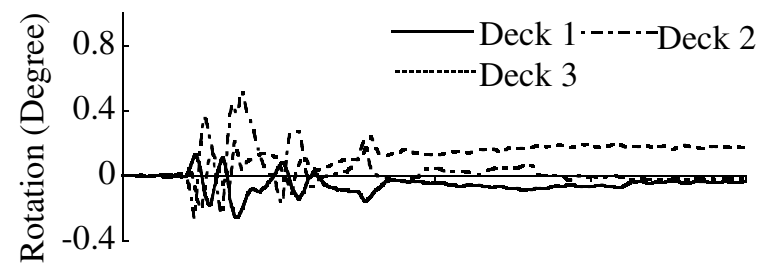

(a) $\Delta_{G}=50 \mathrm{~mm}$

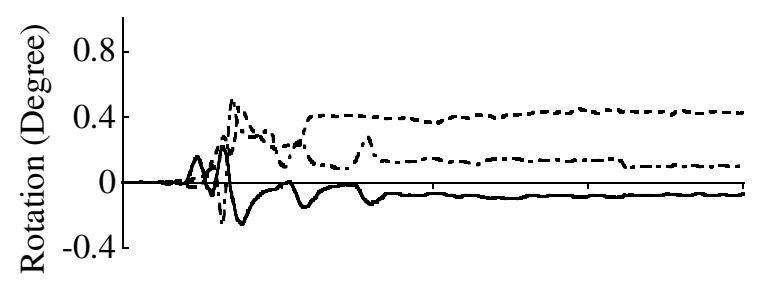

(b) $\Delta_{G}=150 \mathrm{~mm}$

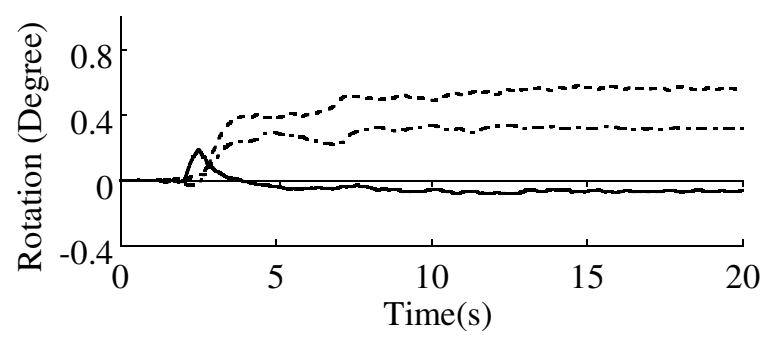

(c) $\Delta_{G}=300 \mathrm{~mm}$

Fig. 12 Effect of Gap Size on Rotation Response of Decks (Type 1 Restrainers)

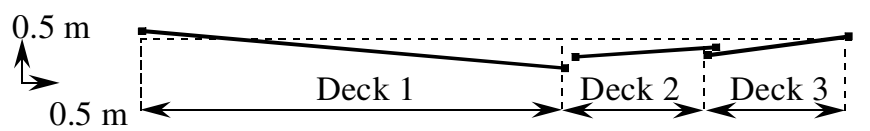

(a) Type 1 Restrainers $(t=3.62 \mathrm{~s})$

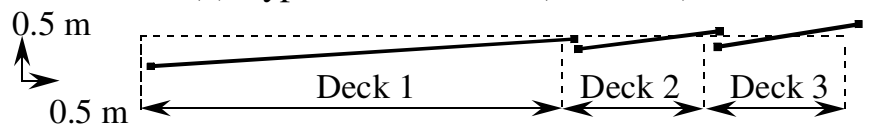

(b) Type 2 Restrainers $(t=3.34 \mathrm{~s})$

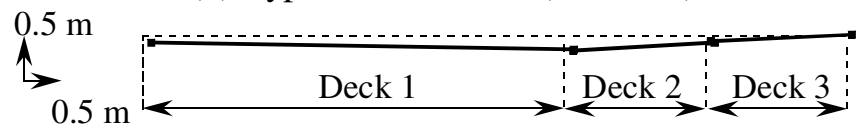

(c) Type 3 Restrainers $(t=3.68 \mathrm{~s})$

Fig. 13 Maximum Displacements of Decks

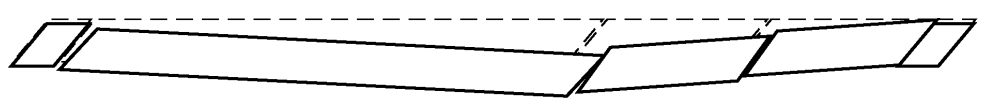

(a) Type 1 and 3 Restrainers

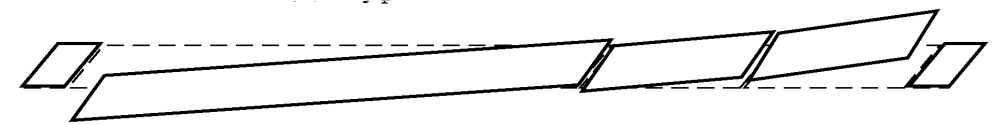

(b) Type 2 Restrainers

Fig.14 Rotations of the Decks 


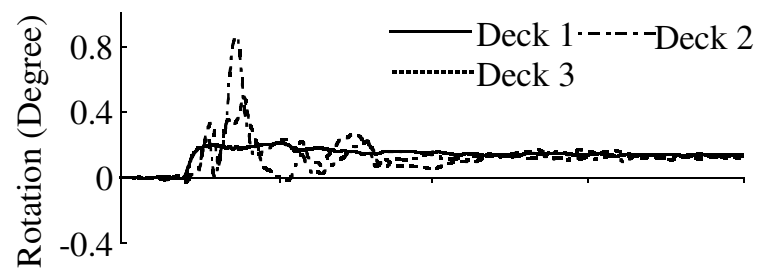

(a) $\Delta_{G}=50 \mathrm{~mm}$

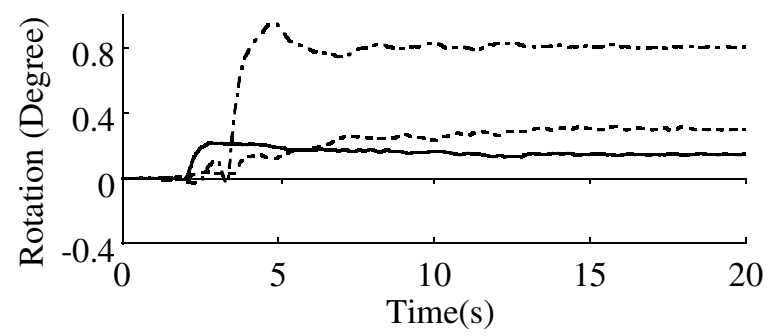

(b) $\Delta_{G}=150 \mathrm{~mm}$

Fig. 15 Effect of Gap Size on Rotation Response of Decks (Type 2 Restrainers)

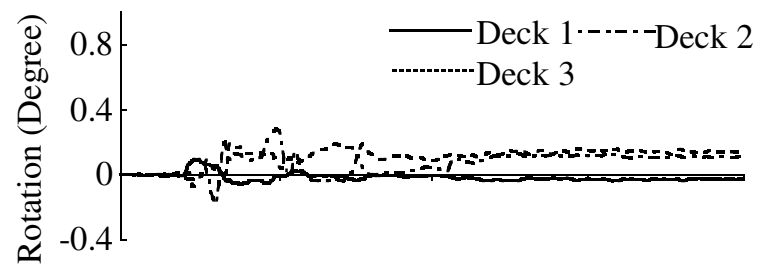

(a) $\Delta_{G}=50 \mathrm{~mm}$

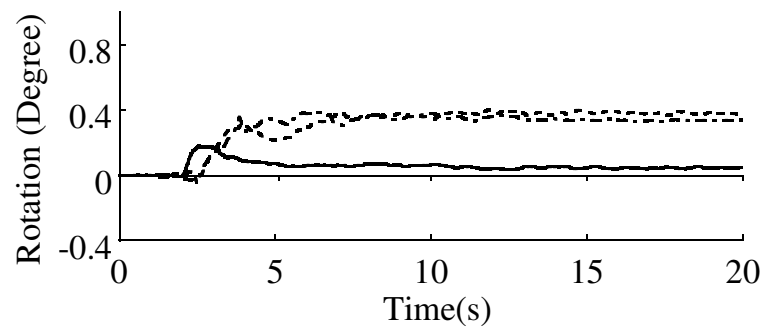

(b) $\Delta_{G}=150 \mathrm{~mm}$

Fig. 16 Effect of Gap Size on Rotation Response of Decks (Type 3 Restrainers)

Fig. 16 shows rotation response of three decks when type 3 restrainers are equipped. The maximum rotations are in the range of -0.2 and 0.2 degree, which is smaller than the maximum rotation of the skewed bridge with type 1 restrainers in thee range of -0.2 and 0.6 degree. This is obviously the effect of restraint of relative displacement by restrainers in both positive and negative directions in the skewed transverse direction. Although the type 2 restrainers do not directly restrict deck relative movements in the longitudinal direction, the displacement of the decks 1, 2 and 3 in the longitudinal direction is 209, 138 and $181 \mathrm{~mm}$, respectively. They are not significantly larger than the displacements of the decks 1,2 and 3 of 117,179 and $110 \mathrm{~mm}$, respectively, when type 2 restrainers are equipped.

It is noted that rotations of the deck in the anticlockwise results in an increase of a distance between adjacent decks at joints as shown in Fig. 17. As a consequence, it is effective from the geometrical 


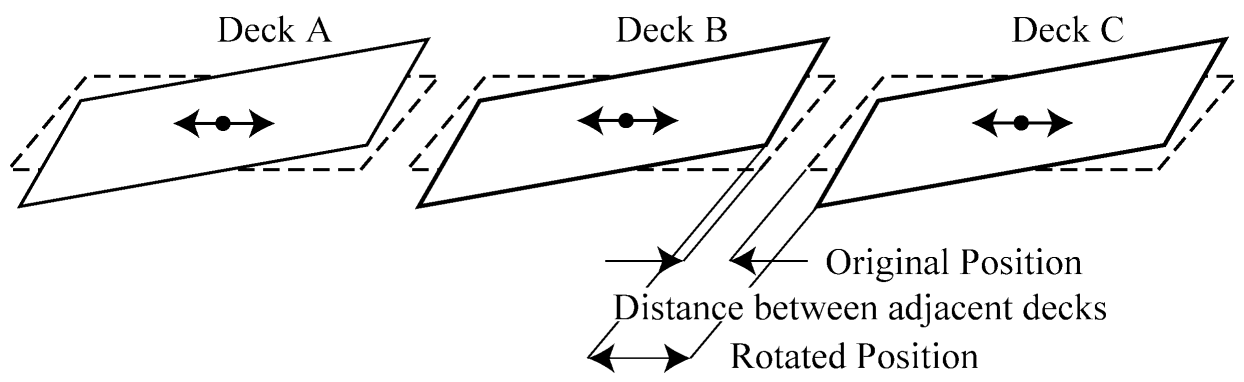

Fig.17 Separation of Adjacent Decks Resulted from Rotations

restriction to limit the deck rotations for controlling the separation between adjacent decks, which, in turn, effective to decrease the risk of unseating the decks from their supports.

As shown in Figs 13 and 14, the displacement mode of the skewed bridge with type 3 restrainers is similar to that with type 1 restrainers.

\section{CONCLUSIONS}

Effect of poundings and cable restrainers was analyzed for a skewed bridge with a skew angle of 50 degree. Three types of restrainers were analyzed. Based on the results presented herein, the following conclusions may be deduced:

1) Poundings of skewed decks to the abutment induce rotations of the decks in a direction that the length supported by the abutment decreases

2) Type 1 restrainers are effective to mitigate the rotations of three decks. This is because the moment induced by type 1 restrainers $M_{R I}$ by Eq. (4) is large enough to pull the deck back to nearly the original position.

3) Type 2 restrainers are less effective than types 1 and 3 restrainers because the moment induced by type 2 restrainers $M_{R 2}$ by Eq. (5) is limited.

4) Type 3 restrainers are effective to directly mitigate the rotation of three decks. It is important to note that it is effective to limit the rotation of three decks for controlling the separation between adjacent decks due to the geometrical constraint.

\section{REFERENCES}

1. Chen, M. C., and Penzien, J. (1975), "Analytical investigations of seismic response of short, single or multiple-span highway bridges," Report No. UCB/EERC 75-4, Earthquake Engineering Research Center, University of California, Berkeley, U.S.A.

2. Liu, W. D., Rieles, J. M., Imbsen, R. A., Priestley, M. J. N., Seible, F., Nobari, F.S. and Yang, R. (1990) "Response of a Major Highway Bridge during the Whitter Earthquake", Proc. 4th U.S. National Conference on Earthquake Engineering, U.S.A.

3. Priestley, M. J. N., Seible, F., and Calvi, G. M. (1995). "Seismic design and retrofit of bridges." Wiley Inter- science, NewYork.

4. Saiidi, M. and Maragakis, E., (1996), "Parameters in bridge restrainer design for seismic retrofit", Journal of Structural Engineering, ASCE, Vol.122, No.19, 61-68. 
5. Trocharkis, P., Eberhard, O. M. and Stanton, F. J. (1997), "Design of seismic restrainers for in-span hinges." Journal of Structural Engineering, ASCE, Vol.123, No.4, 469-478.

6. DesRoches, R. and Fenves, L. G. (2000), "Design of seismic cable hinge restrainers for bridges", Journal of Structural Engineering, ASCE, Vol.126, No.4, 500-509.

7. Kawashima, K. and Shoji, G. (2000) "Effect of restraints to mitigate pounding between adjacent decks subjected to a strong ground motion." 12th WCEE, Paper No. 1435 (CD-ROM), Auckland, New Zealand.

8. Kawashima, K. and Penzien, J. (1979) "Theoretical and experimental dynamic behavior of a curved model bridge structure." Earthquake Engineering and Structural Dynamics, 7, 129-145.

9. Watanabe, G. and Kawashima, K. (2004) "Numerical simulation of poundings of bridges." 13th WCEE, Paper No. 884 (CD-ROM), Vancouver, B.C., Canada. 